\title{
BMJ Open Health-related quality of life and well- being in people over 75 years of age with end-stage kidney disease managed with dialysis or comprehensive conservative care: a cross-sectional study in the UK and Australia
}

Karan K Shah, ${ }^{1}$ Fliss E M Murtagh, ${ }^{2}$ Kevin McGeechan, ${ }^{3}$ Su Crail, ${ }^{4}$ Aine Burns, ${ }^{5}$ Anh D Tran, ${ }^{1}$ Rachael L Morton ${ }^{1}$

To cite: Shah KK, Murtagh FEM, McGeechan K, et al. Healthrelated quality of life and wellbeing in people over 75 years of age with end-stage kidney disease managed with dialysis or comprehensive conservative care: a cross-sectional study in the UK and Australia. BMJ Open 2019;9:e027776. doi:10.1136/ bmjopen-2018-027776

- Prepublication history and additional material for this paper are available online. To view these files, please visit the journal online (http://dx.doi org/10.1136/bmjopen-2018027776).

Received 15 November 2018 Accepted 8 April 2019

Check for updates

(C) Author(s) (or their employer(s)) 2019. Re-use permitted under CC BY-NC. No commercial re-use. See rights and permissions. Published by BMJ.

For numbered affiliations see end of article.

Correspondence to

Karan K Shah;

karan.shah@ctc.usyd.edu.au

\section{ABSTRACT}

Objective To measure health-related quality of life (HRQOL) and well-being in older people with end-stage kidney disease (ESKD) and to determine the association between treatment type and sociodemographic characteristics on these outcome measures. In addition, to assess the convergent validity between the HRQOL and well-being measure and their feasibility and acceptability in this population.

Design Prospective cross-sectional study.

Setting Three renal units in the UK and Australia. Participants 129 patients with ESKD managed with dialysis or with an estimated glomerular filtration $\leq 10 \mathrm{~mL} / \mathrm{min} / 1.73 \mathrm{~m}^{2}$ and managed with comprehensive conservative, non-dialytic care.

Outcome measures $H R Q \mathrm{oL}$ and well-being were assessed using Short-Form six dimensions (SF-6D, 0-1 scale); Kidney Disease Quality of Life (KDQOL-36) (0-100 scale) and Investigating Choice Experiments Capability Measure-Older people (ICECAP-0, 0-1 scale). Linear regression assessed associations between treatment, HRQOL and well-being. Pearson's correlation coefficient assessed convergent validity between instruments.

Results Median age of 81 years (IQR 78-85), 65\% males; $83(64 \%)$ were managed with dialysis and $46(36 \%)$ with conservative care. When adjusted for treatment type and sociodemographic variables, those managed on dialysis reported lower mean SF-6D utility $(-0.05,95 \% \mathrm{Cl}-0.12$ to 0.01); lower KDQOL Physical Component Summary score $(-3.17,95 \% \mathrm{Cl}-7.61$ to 1.27$)$; lower Mental Component Summary score $(-2.41,95 \% \mathrm{Cl}-7.66$ to 2.84$)$; lower quality of life due to burden $(-28.59,95 \% \mathrm{Cl}-41.77$ to $-15.42)$; symptoms $(-5.93,95 \% \mathrm{Cl}-14.61$ to 2.73$)$ and effects of kidney disease $(-16.49,95 \% \mathrm{Cl}-25.98$ to $-6.99)$ and lower overall ICECAP-0 well-being $(-0.07$, $95 \% \mathrm{Cl}-0.16$ to 0.02 ) than those managed conservatively. Correlation between ICECAP-0 well-being and SF-6D utility scores was strong overall, $0.65(p<0.001)$, but weak to moderate at domain level.

Conclusions Older people on dialysis report significantly higher burden and effects of kidney disease than those
Strengths and limitations of this study

The strengths of our study include a prospective assessment of health-related quality of life (HRQoL) in people over 75 years of age and the use of a novel measure to value well-being.

- This information is essential for doctors to discuss the relative benefits of dialysis compared with conservative care.

- The limitation of this study is that the sample size may not have been sufficient to detect a statistically significant difference in mean scores if one existed.

- We did not have complete data on patient's comorbid conditions that may have impacted our ability to explore the associations between comorbid conditions and $\mathrm{HRQOL}$ or well-being.

- Considering the cross-sectional nature of the data, we were unable to analyse any changes relating to individuals' HRQoL or well-being over time, which might be captured in a longitudinal study.

on conservative care. Lower HRQoL and well-being may be associated with dialysis treatment and should inform shared decision-making about treatment options.

Trial registration number UK (IRAS project ID: 134360andREC reference 14/LO/0291) and Australia (R20140203 HREC/14/RAH/36).

\section{INTRODUCTION}

Comprehensive conservative care services were developed for people with end-stage kidney disease (ESKD) in the UK and Australia following the substantial increase in the number of older people aged $\geq 75$ years being referred to nephrologists for dialysis. ${ }^{1}$ Comprehensive conservative care includes interventions to delay the progression of kidney disease and minimise 
complications, as well as detailed communication, shared decision-making, advance care planning and psychologic and family support, but does not include dialysis. ${ }^{2}$ For older patients who often have high levels of comorbidity (such as diabetes and heart disease) and poor functional status, the survival advantage of dialysis may be limited and comprehensive conservative management may be considered; however, robust comparative evidence remains minimal. ${ }^{2}$ Considerations such as symptoms, quality of life and hospital-free days are sometimes more important for patients and families, than expected length of survival. ${ }^{2}$

Traditionally, economists attempt to assist resource allocation decisions by focusing on measuring and valuing health (in its broadest sense), using health-related quality of life (HRQoL) measures and survival, in particular combined in the quality-adjusted life year (QALY). ${ }^{3}$ In QALY calculations, values (often referred to as utility scores) are assigned to different health states, which allows the quantification of health gains comprising both length and quality of life gains from medical interventions. ${ }^{34}$ Utilities are preference weights, where preference can be equated with value or desirability. ${ }^{5}{ }^{6}$ The QALY is then calculated by combining the length of survival and the utility weights.

However, many healthcare interventions may impact more broadly on quality of life (assumed to encompass the broad range of factors that are important to people in living their lives) rather than just health (which centres on physical and mental health). ${ }^{3}$ These broad factors could be related to health and non-health factors that may impact the overall quality of life of a patient. ${ }^{4}$ Measures that look only at health in assessing the impact of these interventions would be very likely to underestimate this impact. ${ }^{37}$

Dialysis has a large impact on the quality of life of both patients and their families; however, traditional HRQoL measures, such as the Short Form 36 (SF-36) and Kidney Disease Quality of Life (KDQOL-36) surveys may be too narrowly focused to detect all of the critical aspects of dialysis that increase or decrease an individual's quality of life. ${ }^{8}$ KDQOL-36 is a short-form questionnaire that includes the SF-12, a generic quality of life questionnaire, ${ }^{910}$ plus disease-specific domains including the burden of kidney disease, symptoms/problems of kidney disease and effects of kidney disease. For this purpose, broader HRQoL measures, often named wellbeing measures, could be used to capture more facets of peoples' lives than health status alone. ${ }^{4}$

New instruments have been developed that provide information across health and social care, rather than just across health. ${ }^{3}$ The recently developed Investigating Choice Experiments Capability Measure (ICECAP) family of instruments have been designed to incorporate such dimensions. ${ }^{11}$ These instruments have their theoretical grounding in Amartya Sen's work on the relationships between functioning and capability. ${ }^{11}{ }^{12}$ They seek to measure a conceptually different evaluative space through a focus on capabilities: that is, what a person is able to do and who they are able to be, rather than on functioning: what a person actually does and who they become. ${ }^{13}$ Capabilities refer to the potential to achieve certain states and perform certain actions. ${ }^{4}$ Having the capability to live life the way one desires is obviously important, also to older people, and reduction of this capability limits their wellbeing. ${ }^{4} 1415$ The ICECAP-O instrument was specifically developed to measure capability in older people. There is little research on how the ICECAP-O is related to other conceptualisations of well-being and the relationships between the ICECAP-O and measures of health (physical, psychological and social) remain underexplored. ${ }^{16}$

The aims of the study were to measure HRQoL using SF-12 questionnaire, KDQoL using KDQOL-36 questionnaire and well-being using ICECAP-O questionnaire (1) to determine the association between treatment type and sociodemographic characteristics on these outcome measures; (2) to assess the convergent validity between the ICECAP-O well-being and the SF-6D utility (derived from SF-12 questionnaire) and (3) to assess the feasibility and acceptability of questionnaires in older patients with ESKD.

\section{MATERIALS AND METHODS \\ Study design}

We conducted a cross-sectional study of patients with ESKD treated with dialysis or comprehensive conservative care in the UK and Australia between 2014 and 2017. The study was performed in accordance with the Australian National Statement on Ethical Conduct in Human Research (2007) and relevant guidance in the UK. The study was reported using Strengthening the Reporting of Observational Studies in Epidemiology guidelines for observational studies (online supplementary item S1). ${ }^{17}$ Eligible subjects were fully informed about the purpose, benefits and risks of the study and signed an approved participant consent form.

\section{Setting and participants}

The study was undertaken at three renal units in the UK and Australia. Included were males and females aged $\geq 75$ years with ESKD, managed with dialysis (facility haemodialysis, home haemodialysis and peritoneal dialysis) or with an estimated glomerular filtration $\leq 10 \mathrm{~mL} / \mathrm{min} / 1.73 \mathrm{~m}^{2}$ and managed with comprehensive conservative, non-dialytic care. The exclusion criteria comprised cognitive impairment; patients unable to read English and patients who were legally blind. To reduce selection bias, nephrologists and clinical nurses in each participating renal unit reviewed their clinic lists for all patients who met the eligibility criteria.

\section{Sample size calculation}

As per the study protocol, a sample size of 194 patients (97 on dialysis, 97 on comprehensive conservative care) 
was calculated to detect a mean difference of 0.05 in the outcomes with $80 \%$ power and $95 \%$ confidence.

\section{Patient and public involvement}

The research question was developed from prior qualitative work with people with ESKD and their carers. ${ }^{18-20}$ Patients were not directly involved in the design of this research study. Patients and their caregivers were informed of the study and invited to participate by the renal unit's research nurses. Participants were provided with an information sheet and consent form for them to read. If they were interested in participating they were asked to sign the consent form and then were provided with two surveys contained in the one booklet (the ICECAP-O survey and the standard KDQOL-36) while at their renal clinic. Patients and their caregivers were assured that participation was voluntary that they did not have to participate and that their decision either way would not affect their clinical care.

\section{Outcomes and variables}

The key outcomes were SF-6D utilities derived from the SF-12 questions, KDQOL scores from the KDQOL-36 questions and ICECAP-O capability index derived from the ICECAP-O questions. Other outcomes were convergent validity between ICECAP-O well-being and the SF-6D utility instrument measured using the Pearson's correlation coefficient; the feasibility and acceptability of the ICECAP-O and SF-12 questionnaires, assessed by response rate and specific items asking the patient whether the questionnaire was easy to complete and whether it covered questions important to their quality of life and well-being.

\section{Data sources/measurement}

All eligible patients were invited to complete the KDQOL-36 (online supplementary item S2) and the five-question ICECAP-O questionnaire (online supplementary item S3) while at their renal clinic. Relevant sociodemographic details such as age, sex, country, educational attainment, private health insurance and questions assessing feasibility and acceptability of the ICECAP-O and SF-12 questionnaire were collected (Item S4). Kidney treatment type (facility haemodialysis, home haemodialysis, peritoneal dialysis and comprehensive conservative care), dialysis status (if currently on dialysis, and time of initiation) and renal transplant status were documented.

\section{HRQoL questionnaire}

The KDQOL-36 has 36 items: the SF-12 version 1 and another 24 kidney-specific items. ${ }^{21}$ The SF-12 responses on the KDQOL-36 were transformed into HRQoL weights, known as utilities, using a published SF-6D algorithm. ${ }^{22}$ The SF-6D is a generic preference-based single measure of health used to generate utilities from six domains: physical, role, social, pain, mental and vital (online supplementary item S5). The SF-6D utilities generated are measured on a 0 (death) to 1 (full health) scale and were reported with mean and SDs using UK population values. $^{22-24}$

The SF-12 section of KDQOL-36 also yields Physical Component Summary (PCS) and Mental Component Summary (MCS) scores, both of which are scored on a T-score metric (mean $=50, \mathrm{SD}=10$, for the US general population). ${ }^{21}{ }^{25}$ The three kidney-specific scales assess burden of kidney disease, symptoms of kidney disease and effects of kidney disease. Each of these scales is scored by transforming all items to a $0-100$ possible range and averaging across the items on each scale to create scale scores. ${ }^{21}$ KDQOL-36 items are all scaled so that higher scores indicate better HRQoL. ${ }^{21} 26$

\section{Well-being questionnaire}

The ICECAP-O questionnaire measures capabilities and covers five domains of well-being, including attachment (love and friendship), security (thinking about the future without concern), role (doing things that make you feel valued), enjoyment (enjoyment and pleasure) and control (independence). ${ }^{27}$ It has four-level response options, representing four levels of capability: none, a little, a lot and all. The responses on the ICECAP-O questions were transformed to a ICECAP-O capability index ranging from 0 (no capability) to 1 (full capability) and presented with mean and SDs using UK population weights. ${ }^{3}$

\section{Quantitative variables}

The SF-6D utilities, KDQOL scores, ICECAP-O capability index and patients' age were treated as continuous, while patients' sex, treatment type (dialysis, conservative care), education (some high school or lower levels, completed high school or higher levels), private health insurance (yes, no) and country (UK, Australia) were analysed as categorical variables. Age was also additionally dichotomised (less than or equal to vs greater than the median age (81 years) $)$.

\section{Statistical methods}

The analysis of data involved descriptive statistics assessing proportions and mean values of the SF-6D utilities, PCS, MCS, Burden of Kidney Disease, Symptoms of Kidney Disease, Effects of Kidney Disease scores and the ICECAP-O capability index for the entire cohort. Hypothesis testing with a two-tailed Student's t-test was used to detect differences in the mean values of SF-6D utilities, KDQOL-36 scores and ICECAP-O capability index for patients' treatment type and sociodemographic characteristics. We hypothesised that HRQoL and well-being measures in each treatment group would be equivalent.

Linear regression with multivariable models was undertaken to determine the association between treatment type and patient characteristics on SF-6D utilities, KDQOL scores and ICECAP-O capability index. In the multivariable linear regression, age, sex, treatment type, education, private health insurance and country were included as covariates on the basis of a priori knowledge of their associations with the HRQoL and well-being measures. 
Pearson's correlation coefficient was used to determine the convergent validity of the ICECAP-O well-being with the SF-6D utility instrument. The correlations were assessed for the overall ICECAP-O and SF-6D utility scores and their domains. We hypothesised, moderate to strong positive correlations because both these instruments measures some similar facets of quality of life. Correlations above 0.5 were considered strong, between 0.3 and 0.5 as moderate and below 0.3 as weak. ${ }^{16}$

Complete case analysis was performed for all outcomes. All statistical analyses were performed with SAS V.9.4. A $p$ value of $<0.05$ was considered statistically significant.

\section{RESULTS}

A total of 129 patients were recruited, including 83 (64\%) managed with dialysis and $46(36 \%)$ patients managed with comprehensive conservative care. The majority of conservatively treated patients were from Australia $(n=37)$ and most treated with dialysis were from the UK $(n=58)$. Overall, $65 \%$ were male, and the median age of the entire cohort was 81 years (IQR 78-85). Patient characteristics are shown in table 1 .

\section{HRQoL SF-6D utilities}

Of 129 patients, the mean utility for 116 patients with complete data was 0.62 (SD 0.14) ( $\mathrm{n}=13$ missing values). The mean SF-6D utilities for the dialysis group were 0.61 (SD 0.13) and 0.65 (SD 0.15) for the conservative care group (online supplementary table S1). The 'vitality' domain reported the highest average score and was responsible for the highest decrement in utilities in both treatment groups (online supplementary table S2).

The mean SF-6D utilities were 0.07 (SD 0.14) lower for females than for males $(p=0.006) ; 0.06$ (SD 0.14) lower for patients residing in the UK compared with those residing in Australia ( $\mathrm{p}=0.03$ ) and 0.07 (SD 0.14) lower for patients without a private health insurance compared to patients with a private health insurance $(\mathrm{p}=0.03)$ (online supplementary table $\mathrm{S} 1$ ). When adjusted for all variables, the mean SF-6D utilities were 0.09 lower for females compared with males $(95 \%$ lower $\mathrm{CI}=-0.14$ and upper

Table 1 Patients characteristics according to treatment group

\begin{tabular}{|c|c|c|c|}
\hline & Dialysis & Conservative Care & Total \\
\hline & $n=83$ & $\mathrm{n}=46$ & $n=129$ \\
\hline Patient Characteristics & n (\%) & n (\%) & n (\%) \\
\hline \multicolumn{4}{|l|}{ Dialysis } \\
\hline Facility haemodialysis & $68(82$ & - & $68(53)$ \\
\hline Home haemodialysis & $2(2)$ & - & $2(2)$ \\
\hline Peritoneal dialysis & $13(16)$ & - & $13(10)$ \\
\hline Median age (years) & $81(78-84)$ & $83(81-87)$ & $81(78-85)$ \\
\hline \multicolumn{4}{|l|}{ Age group } \\
\hline$\leq 81$ years & $50(60)$ & $19(41)$ & $69(53)$ \\
\hline$>81$ years & $33(40)$ & $27(59)$ & $60(47)$ \\
\hline \multicolumn{4}{|l|}{ Sex } \\
\hline Males & $57(69)$ & $27(59)$ & $84(65)$ \\
\hline Females & $26(31)$ & $19(41)$ & $45(35)$ \\
\hline \multicolumn{4}{|l|}{ Country } \\
\hline UK & $58(70)$ & $9(20)$ & $67(52)$ \\
\hline Australia & $25(30)$ & $37(80)$ & $62(48)$ \\
\hline \multicolumn{4}{|l|}{ Education } \\
\hline Primary school & $26(31)$ & $19(41)$ & $45(35)$ \\
\hline Some high school & $35(42)$ & $17(37)$ & $52(40)$ \\
\hline Completed high school & $8(10)$ & $3(7)$ & $11(9)$ \\
\hline Completed diploma & $6(7)$ & $3(7)$ & $9(7)$ \\
\hline Completed university degree & $7(8)$ & $3(7)$ & $10(8)$ \\
\hline \multicolumn{4}{|l|}{ Private health insurance } \\
\hline Yes & $15(18)$ & $14(30)$ & $29(22)$ \\
\hline No & $65(78)$ & $29(63)$ & $94(73)$ \\
\hline Unknown & $1(1)$ & $1(2)$ & $2(2)$ \\
\hline
\end{tabular}


Table 2 Adjusted difference in SF-6D utilities, KDQOL-36 scores and ICECAP-O capability index for dialysis compared with conservative care (fully adjusted)

\begin{tabular}{lcccc}
\hline & Differences & 95\% lower $\mathbf{C l}$ & 95\% upper Cl & P value \\
\hline SF-6D utilities & -0.05 & -0.12 & 0.01 & 0.12 \\
KDQOL-PCS & -3.17 & -7.61 & 1.27 & 0.16 \\
KDQOL-MCS & -2.41 & -7.66 & 2.84 & 0.37 \\
KDQOL-burden of disease & -28.59 & -41.77 & -15.42 & $<0.001 \dagger$ \\
KDQOL-symptoms of disease & -5.93 & -14.61 & 2.73 & 0.18 \\
KDQOL-effects of disease & -16.49 & -25.98 & -6.99 & $<0.001 \dagger$ \\
ICECAP-O capability index & -0.07 & -0.16 & 0.02 & 0.12 \\
\hline
\end{tabular}

${ }^{*}$ Difference in scores adjusted for age, gender, country, education and health insurance status.

$\dagger P<0.001$, statistical significance.

ICECAP-O, Investigating Choice Experiments Capability Measure- Older people; KDQOL-36, Kidney Disease Quality of Life with 36 items; MCS, mental component summary; PCS, physical component summary; SF-6D, Short-Form six dimensions.

$\mathrm{CI}=-0.03, \mathrm{p}=0.002)$. There was no significant difference in the mean utilities observed between two treatments when adjusted for other variables (table 2).

\section{KDQOL scores}

The mean KDQOL scores on the five domains for patients with complete data were as follows: PCS score of 32.41 ( $\mathrm{n}=115$, SD 9.68); MCS score of 47.25 ( $\mathrm{n}=115$, SD 11.34); Burden of Kidney Disease score of 44.46 ( $n=127$, SD 31.28); Symptom/Problems of Kidney Disease score of 72.78 ( $\mathrm{n}=125, \mathrm{SD} 19.03)$ and Effects of Kidney Disease score of 70.24 ( $n=127$, SD 22.35).

In univariate analysis, the PCS score was 5.46 points lower in females than males $(\mathrm{p}=0.004)$ (ie, lower physical health); the MCS score was 4.63 points lower in Australian versus UK patients $(\mathrm{p}=0.03$ ) (ie, lower mental health) (online supplementary table S1 and table S3). The Burden of Kidney Disease score was 28.12 points lower in the dialysis group than the conservative care group $(p<0.001)$ (indicating a higher burden of disease

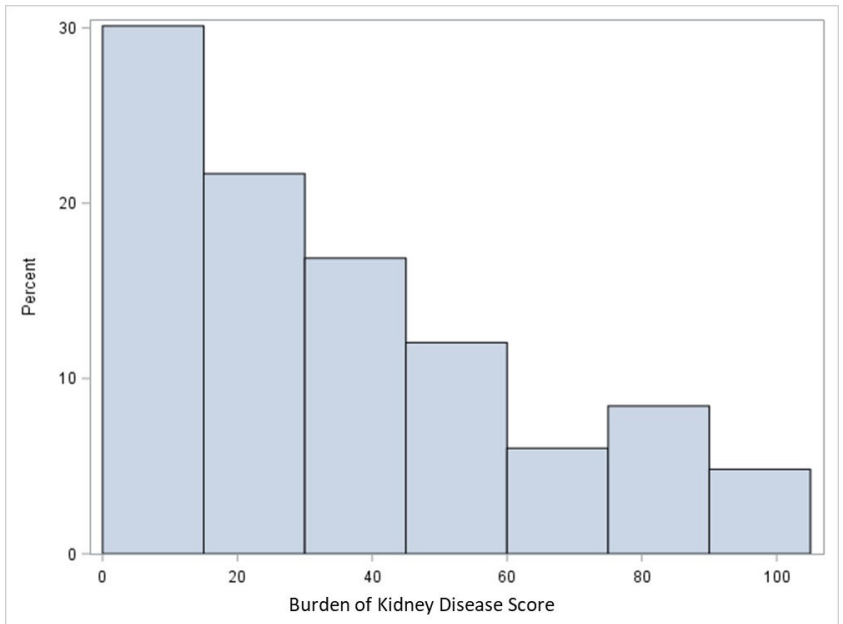

Figure 1 Kidney Disease Quality of Life-36 Burden of Kidney Disease score for dialysis group $(n=83)$. A higher score indicates lower burden of disease and better quality of life. and lower quality of life) (figures 1 and 2); 14.06 points lower in UK versus Australian patients $(\mathrm{p}=0.01)$ (indicating higher burden of disease); 13.70 points lower in patients without private health insurance compared with those with private health insurance $(\mathrm{p}=0.04)$ (indicating a higher burden of disease). The Effects of Kidney Disease score was 17.11 points lower in the dialysis group compared with the conservative care group $(p<0.001)$ (indicating higher effects of the disease and lower quality of life) (figures 3 and 4); 8.35 points lower in UK versus Australian patients $(p=0.03)$ (indicating higher effects of the disease).

The dialysis group reported a higher MCS score (47.67 vs 46.56), indicating marginally better mental health than the conservative care group (online supplementary table S2).

When adjusted for other variables, the mean score for the burden of kidney disease subscale was 28.59 lower (ie, more burdensome) for patients on dialysis compared with patients on conservative care $(\mathrm{p}<0.001)$

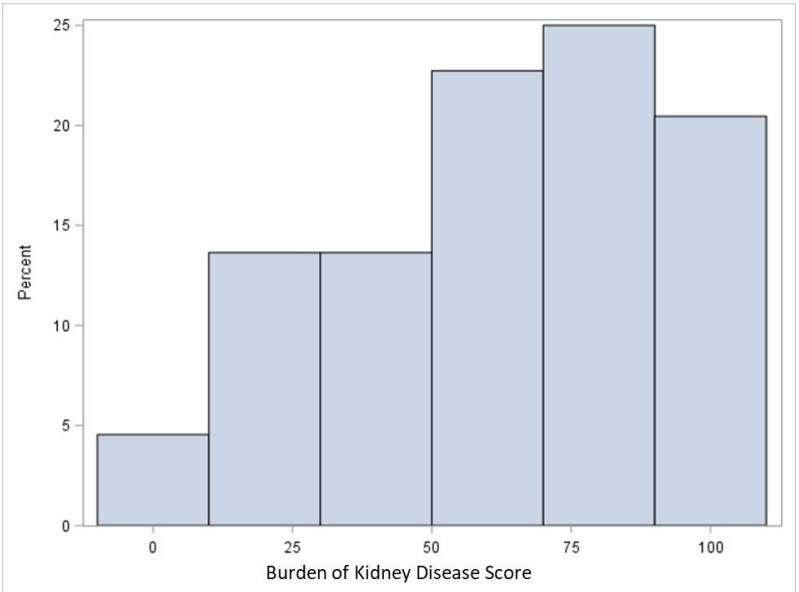

Figure 2 Kidney Disease Quality of Life-36 Burden of Kidney Disease score for conservative care group $(n=44)$. A higher score indicates lower burden of disease and better quality of life. 


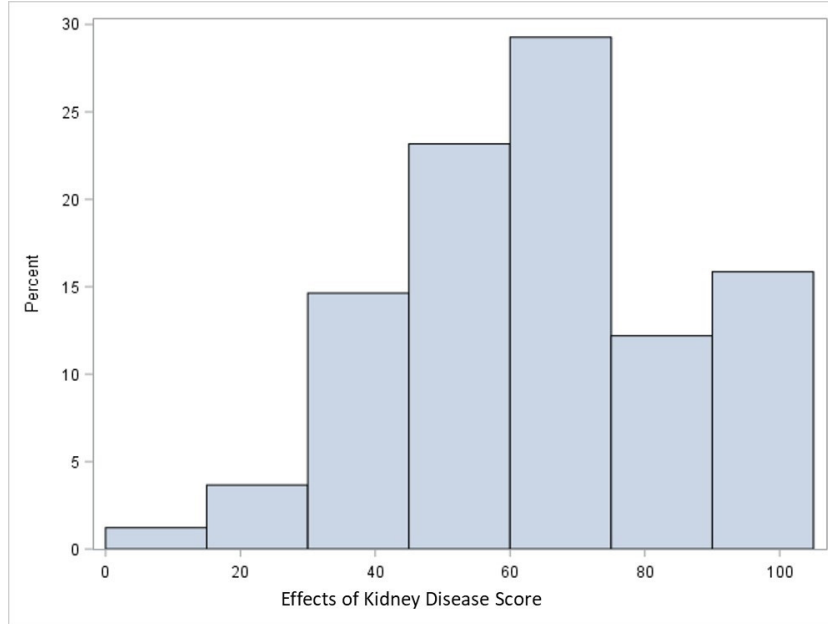

Figure 3 Kidney Disease Quality of Life-36 Effects of Kidney Disease score for dialysis group $(n=82)$. A higher score indicates lower effects of disease and better quality of life.

(table 2). The mean score for effects of kidney disease when adjusted for all the other variables, was 16.49 lower (ie, higher disease-related effects) for patients on dialysis compared with patients on comprehensive conservative care $(\mathrm{p}<0.001)$ (table 2). Adjusted scores were lower but not statistically, significantly different for PCS, MCS and symptoms of kidney disease between the two treatment groups.

\section{ICECAP-0 capability index}

The mean ICECAP-O capability index for 126 patients with complete data was 0.72 (SD 0.19) ( $\mathrm{n}=3$ missing values). In the dialysis group, the mean capability index was 0.71 (SD 0.19 ) and 0.76 (SD 0.20) for the conservative care group (online supplementary table S1), but not significantly different. Overall, the dialysis treatment group reported a lower well-being score on all five domains compared with the conservative care group. The 'attachment' domain

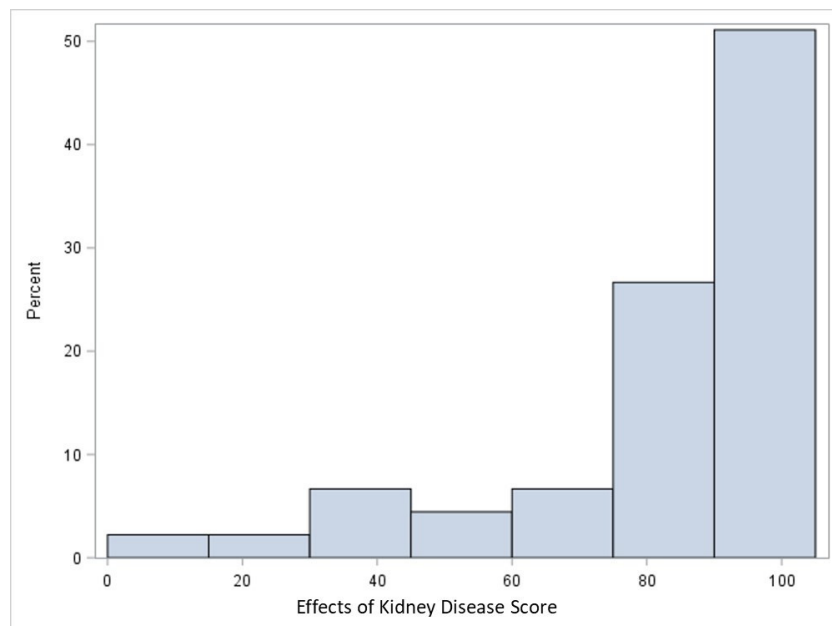

Figure 4 Kidney Disease Quality of Life-36 Effects of Kidney Disease score for conservative care group $(n=45)$. A higher score indicates lower effects of disease and better quality of life. showed the highest average score and was responsible for the highest contribution to capabilities in both treatment groups (online supplementary table S2). When adjusted for other variables, there were no significant differences in the mean capability index observed between the two treatments (table 2).

\section{Convergent validity}

For 114 observations, the overall SF-6D utilities and the pain domain of the SF-6D were strongly correlated with the overall ICECAP-O capability index with a Pearson's coefficient of $0.65(\mathrm{p}<0.001)$ and $0.56(\mathrm{p}<0.001)$, respectively. At the domain level, the role and control domains of the ICECAP-O questionnaire were strongly correlated with the pain domain of the SF-6D, with a Pearson's coefficient of $0.51(\mathrm{p}<0.001)$ and $0.53(\mathrm{p}<0.001)$, respectively. All other domains of the ICECAP-O were weakly or moderately correlated with SF-6D domains, values ranging from 0.02 to 0.49 (table 3 ).

\section{Feasibility and acceptability}

One hundred and fifteen of 129 patients completed the questionnaire, with 14 patients missing items for the ICECAP-O and 10 patients missing items for the SF-12. Overall, patients found both questionnaires easy to use and relevant to assessing their well-being. They responded with an average score of 1.78 out of 5 ( 1 =strongly agree, $5=$ completely disagree) on questions assessing ease of use and with an average score 1.77 and 1.79 out of 5 on the questions assessing the relevance of ICECAP-O and the SF-12 questions, respectively.

\section{DISCUSSION}

This prospective cross-sectional study determined the mean SF-6D utilities, KDQOL scores and ICECAP-O capability index for patients with ESKD according to treatment and sociodemographic variables. Our findings suggest females compared with males, patients residing in the UK compared with those residing in Australia and patients without private health insurance compared with those with private health insurance have significantly lower SF-6D utilities. However, when adjusted for the other variables, only females reported significantly lower utilities compared with males. Furthermore, the study determined the convergent validity between the ICECAP-O well-being and SF-6D utility instrument and assessed the feasibility and acceptability of the ICECAP-O well-being and SF-12 questionnaire in older people with ESKD.

The dialysis group reported 0.05 lower SF-6D utilities compared with the conservative care group reflecting a potentially clinically meaningful difference related to treatment; however, this difference was not statistically significant. Meaningful differences or the minimal important difference (MID) in utility-based HRQoL reported in 11 studies using the SF-6D utilities ranged from 0.011 to 0.097 , with a mean MID of $0.041 .^{28}$ It is therefore likely our study has detected a meaningful difference. In 
Table 3 Convergent validity between ICECAP-O and SF-6D measures $(n=114) \dagger$ using Pearson's correlation coefficient

\begin{tabular}{|c|c|c|c|c|c|c|}
\hline & $\begin{array}{l}\text { ICECAP-O } \\
\text { overall }\end{array}$ & $\begin{array}{l}\text { ICECAP-O } \\
\text { domain } \\
\text { attachment }\end{array}$ & Security & Role & Enjoyment & Control \\
\hline SF-6D overall & $0.65^{\star *}$ & - & - & - & - & - \\
\hline \multicolumn{7}{|l|}{ SF-6D domain } \\
\hline Physical health & $0.43^{\star *}$ & 0.08 & $0.31^{*}$ & $0.40^{* *}$ & $0.32^{*}$ & $0.40^{* *}$ \\
\hline Role limitations & $0.30^{*}$ & 0.05 & $0.21^{*}$ & $0.28^{\star}$ & 0.14 & $0.31^{*}$ \\
\hline Social functioning & $0.41^{* *}$ & 0.18 & $0.25^{*}$ & $0.34^{*}$ & $0.30^{*}$ & $0.35^{*}$ \\
\hline Pain & $0.56^{\star *}$ & 0.17 & $0.29^{*}$ & $0.51^{\star *}$ & $0.43^{\star \star}$ & $0.53^{\star \star}$ \\
\hline Mental health & $0.39^{* *}$ & $0.19^{*}$ & $0.35^{*}$ & $0.30^{*}$ & $0.27^{*}$ & $0.27^{*}$ \\
\hline Vitality & $0.44^{\star \star}$ & 0.17 & $0.21^{*}$ & $0.41^{\star \star}$ & $0.28^{\star}$ & $0.42^{\star \star}$ \\
\hline
\end{tabular}

${ }^{*} \mathrm{P}<0.05 ;{ }^{* *} \mathrm{P}<0.001$

†Observations with missing values on either SF-12 or ICECAP-O questions were removed from the analysis $(n=15)$.

ICECAP-O, Investigating Choice Experiments Capability Measure-Older people; SF-6D, Short-Form six dimensions; SF-12, Short-Form 12 dimensions.

addition, a 0.05 difference in ICECAP-O well-being for dialysis patients may also represent a clinically meaningful difference; however, MIDs for ICECAP-O have not yet been published. Similarly, the KDQOL-36 instrument identified a higher burden of disease and greater effects of the disease for those on dialysis. This finding needs to be explored further in a larger sample size to investigate the potential detrimental effects of dialysis on HRQoL.

In our study, with the exception of a strong correlation between the 'control' and 'role' domain of the ICECAP-O with the 'pain' domain on the SF-6D, most of the ICECAP-O domains were found to have weak to moderate correlations with the SF-6D corresponding domains. This indicates that the newly developed capability instrument does measure different aspects of quality of life or wellbeing and offers additional information when compared with measures of health, such as the SF-6D used in the conventional QALY approach. In addition, we observed a higher score for the feasibility and acceptability of the ICECAP-O questions indicating it to be acceptable and as relevant as SF-12 (an established HRQoL measure).

There is debate in the health economics literature concerning the ways to apply the capability approach in economic evaluations with some suggesting that QALYs alone are adequate, while others argue this approach is too narrow and that direct measures of capability or well-being provide a more extensive application of Sen's paradigm. ${ }^{29}$ Capability is empirically distinct from functioning and the content of capability instruments is not subsumed by the content of instruments used to capture changes in HRQoL for QALYs. ${ }^{29}$

Health economic analyses would benefit from the inclusion of individual capability measures; whether the focus should be only on people's achievements - their 'functioning'-or people's capability to achieve is contested. ${ }^{29}$ Sen's example of the fasting man versus the starving man serves as a key example for focusing on capability: two people, one of whom is starving and the other, who is fasting, have comparable functioning in terms of nourishment, but their capabilities to be nourished are notably different. ${ }^{29}$ The argument is that focusing on functioning alone would miss important distinctions, such as differences in freedom and choice between individuals. ${ }^{29}$

There are some limitations to this study. First, we were only able to recruit 129 of the 194 patients outlined in the protocol sample size, as some of the study sites were unable to participate. Hence, the sample size may not have been sufficient to detect a statistically significant difference in mean scores if one existed. Second, our observational study of older patients with ESKD may not have perfectly matched the two groups with respect to co-morbid conditions or rate of renal decline. We did not have complete data on comorbidities and this may have impacted our ability to explore the associations between treatment type, HRQoL or well-being. Third, considering the cross-sectional nature of the data, we were unable to analyse any changes relating to individuals' HRQoL or well-being over time, which might be captured in a longitudinal study. The strengths of our study include a prospective assessment of HRQoL in people over 75 years of age and the use of a novel measure to value well-being. This information is essential for doctors to discuss the relative benefits of dialysis compared with conservative care.

In conclusion, we observed lower quality of life and well-being for older patients with ESKD managed on dialysis compared with comprehensive conservative care. Furthermore, measuring well-being using a capability index provides additional insights into the impact of dialysis on older people than HRQoL measurement alone and has potential to improve the economic evaluation of treatment for ESKD.

Author affiliations

${ }^{1}$ Health Economics, The University of Sydney, NHMRC Clinical Trials Centre, Sydney, New South Wales, Australia 
${ }^{2}$ Wolfson Palliative Care Research Centre, Hull York Medical School, University of Hull, Hull, UK

${ }^{3}$ Biostatistics, School of Public Health, The University of Sydney, Sydney, New South Wales, Australia

${ }^{4}$ Central Northern Adelaide Renal and Transplantation Service, Royal Adelaide Hospital, Adelaide, South Australia, Australia

${ }^{5}$ Nephrology, Royal Free Hospital, London, UK

Acknowledgements The authors gratefully acknowledge the patients who participated in this study; the research nurses from the UK and Australian renal units and Dr Sherilyn Goldstone for proof reading the final version of the manuscript.

Contributors FEMM, KM, SC, AB and RLM designed the study. SC, AB and RLM led the data collection. KKS conducted the analysis and drafted the first version of the manuscript. KM, ADT and RLM supported the data analysis and interpretation of the results. All authors revised the final version of the manuscript.

Funding This work was supported by the NHMRC Early Career Researcher Fellowship and Sidney Sax - Public Health Overseas Fellowship (1054216) grant awarded to Rachael Morton for the conduct of the ICECAP-0 study. Karan Shah is employed at the NHMRC Clinical Trials Centre, University of Sydney, and is fully funded by the institution. The remaining authors declare that they have no other relevant financial interests.

Patient consent for publication Not required.

Ethics approval The study was performed inaccordance with the NHMRC National Statement on Ethical Conduct in HumanResearch (Commonwealth of Australia, 2007), and relevant guidance in the UK.Each renal unit participating in the study obtained the approval of the InstitutionalHealth Research Ethics Committee to conduct the study. The study approvalnumbers are as follow: UK (IRAS project ID: 134360 \& REC reference14/L0/0291) and Australia (R20140203 HREC/14/RAH/36)

Provenance and peer review Not commissioned; externally peer reviewed.

Data sharing statement Data for the study can be provided for specific research questions that are available from the corresponding author on request.

Open access This is an open access article distributed in accordance with the Creative Commons Attribution Non Commercial (CC BY-NC 4.0) license, which permits others to distribute, remix, adapt, build upon this work non-commercially, and license their derivative works on different terms, provided the original work is properly cited, appropriate credit is given, any changes made indicated, and the use is non-commercial. See: http://creativecommons.org/licenses/by-nc/4.0/.

\section{REFERENCES}

1. Morton RL, Turner RM, Howard K, et al. Patients who plan for conservative care rather than dialysis: a national observational study in Australia. Am J Kidney Dis 2012;59:419-27.

2. Murtagh FE, Burns A, Moranne O, et al. Supportive care: comprehensive conservative care in end-stage kidney disease. Clin J Am Soc Nephrol 2016;11:1909-14.

3. Coast J, Flynn TN, Natarajan L, et al. Valuing the ICECAP capability index for older people. Soc Sci Med 2008;67:874-82.

4. Makai P, Beckebans F, van Exel J, et al. Quality of life of nursing home residents with dementia: validation of the German version of the ICECAP-O. PLOS One 2014;9:e92016.

5. Weinstein MC, Torrance G, McGuire A. QALYs: the basics. Value Health 2009;12:S5-S9.

6. Whitehead SJ, Ali S. Health outcomes in economic evaluation: the QALY and utilities. Br Med Bull 2010;96:5-21.
7. Walker RC, Howard K, Tong A, et al. The economic considerations of patients and caregivers in choice of dialysis modality. Hemodial Int 2016;20:634-42.

8. Murtagh FE, Marsh JE, Donohoe P, et al. Dialysis or not? A comparative survival study of patients over 75 years with chronic kidney disease stage 5. Nephrol Dial Transplant 2007;22:1955-62.

9. Ware J, Kosinski M, Keller SD. A 12-Item Short-Form Health Survey: construction of scales and preliminary tests of reliability and validity. Med Care 1996;34:220-33.

10. Wyld ML, Chadban SJ, Morton RL. Improving Our Understanding of Quality of Life in CKD. Am J Kidney Dis 2016;67:820-1.

11. Chen G, Ratcliffe J, Kaambwa B, et al. Empirical Comparison Between Capability and Two Health-Related Quality of Life Measures. Soc Indic Res:1-16.

12. Al-Janabi H, Flynn TN, Coast J. Development of a self-report measure of capability wellbeing for adults: the ICECAP-A. Qual Life Res 2012;21:167-76.

13. Coast J, Smith RD, Lorgelly P. Welfarism, extra-welfarism and capability: the spread of ideas in health economics. Soc Sci Med 2008;67:1190-8.

14. Sen A. Choice, welfare, and measurement: Harvard University Press, 1997.

15. Grewal I, Lewis J, Flynn T, et al. Developing attributes for a generic quality of life measure for older people: preferences or capabilities? Soc Sci Med 2006;62:1891-901.

16. Makai P, Koopmanschap MA, Brouwer WB, et al. A validation of the ICECAP-O in a population of post-hospitalized older people in the Netherlands. Health Qual Life Outcomes 2013;11:57.

17. Vandenbroucke JP, von Elm E, Altman DG, et al. Strengthening the Reporting of Observational Studies in Epidemiology (STROBE): explanation and elaboration. PLoS Med 2007:4:e297.

18. Sellars M, Clayton JM, Morton RL, et al. An Interview Study of Patient and Caregiver Perspectives on Advance Care Planning in ESRD. Am J Kidney Dis 2018;71:216-24.

19. Walker RC, Howard K, Morton RL, et al. Patient and caregiver values, beliefs and experiences when considering home dialysis as a treatment option: a semi-structured interview study. Nephrol Dial Transplant 2016;31:133-41.

20. Morton RL, Tong A, Webster AC, et al. Characteristics of dialysis important to patients and family caregivers: a mixed methods approach. Nephrol Dial Transplant 2011;26:4038-46.

21. Peipert JD, Bentler PM, Klicko K, et al. Psychometric Properties of the Kidney Disease Quality of Life 36-Item Short-Form Survey (KDQOL-36) in the United States. Am J Kidney Dis 2018;71:461-8.

22. Brazier JE, Roberts J. The estimation of a preference-based measure of health from the SF-12. Med Care 2004:42:851-9.

23. Brazier J, Roberts J, Deverill M. The estimation of a preferencebased measure of health from the SF-36. $J$ Health Econ 2002;21:271-92.

24. Kharroubi SA, Brazier JE, Roberts J, et al. Modelling SF-6D health state preference data using a nonparametric Bayesian method. $J$ Health Econ 2007;26:597-612.

25. Ware JE, Keller SD, Kosinski M. SF-12: How to score the SF-12 physical and mental health summary scales: Health Institute. New England Medical Center 1995.

26. Hays R, Spritzer K. KDQOL-36 ${ }^{\mathrm{TM}}$ Scoring Program (v1. 0), 2000.

27. Flynn TN, Chan P, Coast J, et al. Assessing quality of life among British older people using the ICEPOP CAPability (ICECAP-O) measure. Appl Health Econ Health Policy 2011;9:317-29.

28. Walters SJ, Brazier JE. Comparison of the minimally important difference for two health state utility measures: EQ-5D and SF-6D. Qual Life Res 2005;14:1523-32.

29. Mitchell PM, Venkatapuram S, Richardson J, et al. Are qualityadjusted life years a good proxy measure of individual capabilities? Pharmacoeconomics 2017;35:637-46. 